Distinct differences in rates of oxygen consumption and ATP synthesis of regionally isolated non-synaptic mouse brain mitochondria

Andersen, Jens V; Jakobsen, Emil; Waagepetersen, Helle S; Aldana, Blanca I

Published in:

Journal of Neuroscience Research

DOI:

10.1002/jnr.24371

Publication date:

2019

Document version

Publisher's PDF, also known as Version of record

Document license:

CC BY

Citation for published version (APA):

Andersen, J. V., Jakobsen, E., Waagepetersen, H. S., \& Aldana, B. I. (2019). Distinct differences in rates of oxygen consumption and ATP synthesis of regionally isolated non-synaptic mouse brain mitochondria. Journal of Neuroscience Research, 97, 961-974. https://doi.org/10.1002/jnr.24371 


\section{gibco}

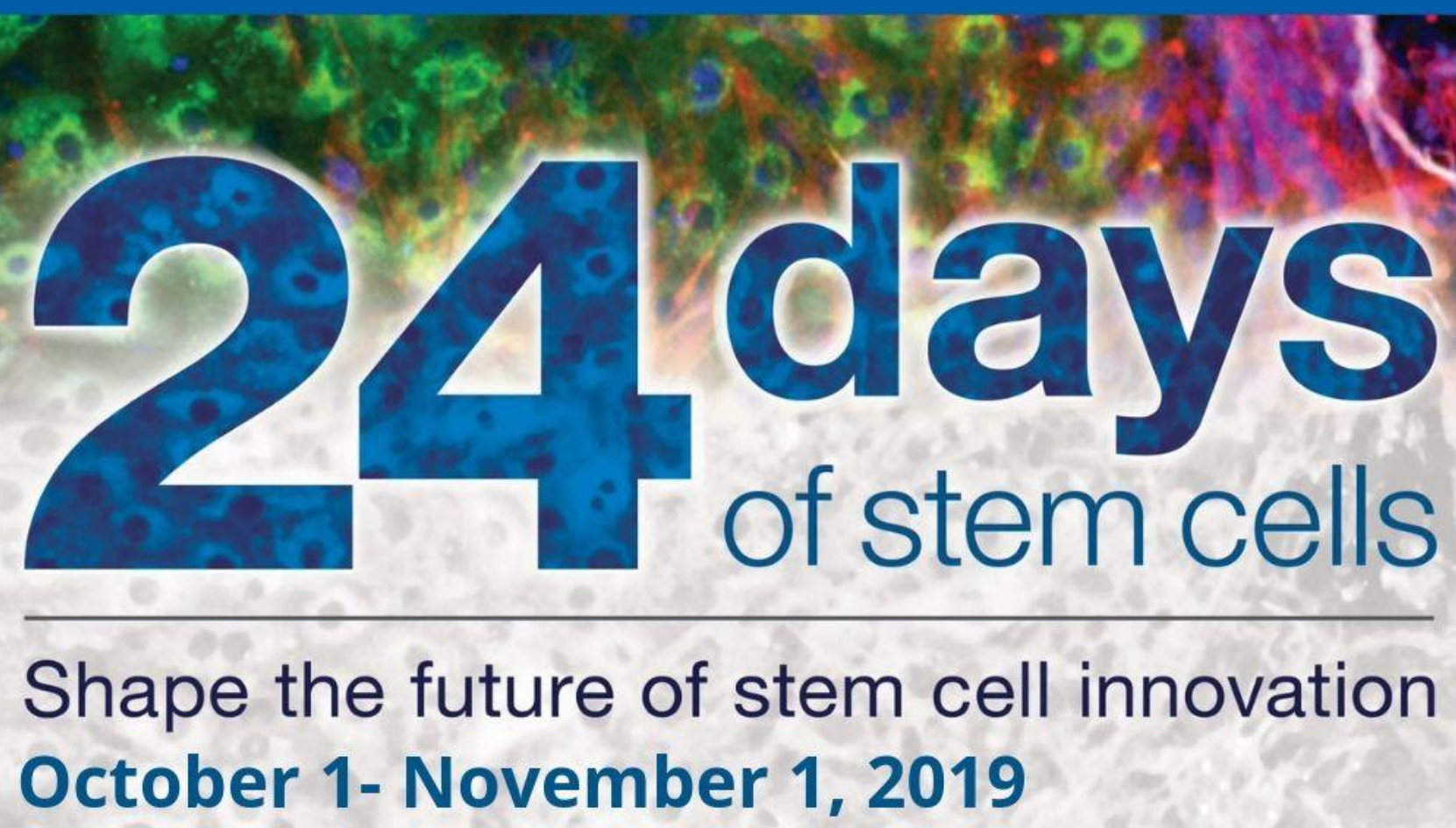

Join us for 24 Days of Stem Cells; a premiere virtual event featuring the latest advances in stem cell research.

This year's format will feature a new hour of cutting edge content every week day starting October 1st. Attend the sessions that are most relevant to your work - at your convenience and at your pace.

During the 24-day long event, you can:

- Access leading scientific presentations from thought leaders around the world

- Watch live training demonstrations from our stem cell experts

- Download key stem cell tools and resources

- Complete weekly challenges to earn points towards certification and prizes

Register today at

ThermoFisher www.24daysofstemcells.com S C I E N T I F I C 


\title{
Distinct differences in rates of oxygen consumption and ATP synthesis of regionally isolated non-synaptic mouse brain mitochondria
}

\author{
Jens V. Andersen (D) | Emil Jakobsen (iD | Helle S. Waagepetersen (iD | \\ Blanca I. Aldana
}

Faculty of Health and Medical Sciences, Department of Drug Design and Pharmacology, University of Copenhagen, Copenhagen, Denmark

\section{Correspondence}

Jens V. Andersen and Blanca I. Aldana, Faculty of Health and Medical Sciences, Department of Drug Design and Pharmacology, University of Copenhagen, Universitetsparken 2, Copenhagen 2100, Denmark.

Emails: Jens.Andersen@sund.ku.dk; Blanca. Aldana@sund.ku.dk

\begin{abstract}
Brain mitochondrial dysfunction has been implicated in several neurodegenerative diseases. The distribution and efficiency of mitochondria display large heterogeneity throughout the regions of the brain. This may imply that the selective regional susceptibility of neurodegenerative diseases could be mediated through inherent differences in regional mitochondrial function. To investigate regional cerebral mitochondrial energetics, the rates of oxygen consumption and adenosine- 5 '-triphosphate (ATP) synthesis were assessed in isolated non-synaptic mitochondria of the cerebral cortex, hippocampus, and striatum of the male mouse brain. Oxygen consumption rates were assessed using a Seahorse XFe96 analyzer and ATP synthesis rates were determined by an online luciferin-luciferase coupled luminescence assay. Complex I- and complex II-driven respiration and ATP synthesis, were investigated by applying pyruvate in combination with malate, or succinate, as respiratory substrates, respectively. Hippocampal mitochondria exhibited the lowest basal and adenosine-5'-diphosphate (ADP)-stimulated rate of oxygen consumption when provided pyruvate and malate. However, hippocampal mitochondria also exhibited an increased proton leak and an elevated relative rate of oxygen consumption in response to the uncoupler carbonyl cyanide 4-(trifluoromethoxy)phenylhydrazone (FCCP), showing a large capacity for uncoupled respiration in the presence of pyruvate. When the complex II-linked substrate succinate was provided, striatal mitochondria exhibited the highest respiration and ATP synthesis rate, whereas hippocampal mitochondria had the lowest. However, the mitochondrial efficiency, determined as ATP produced $/ \mathrm{O}_{2}$ consumed, was similar between the three regions. This study reveals inherent differences in regional mitochondrial energetics and may serve as a tool for further investigations of regional mitochondrial function in relation to neurodegenerative diseases.
\end{abstract}

KEYWORDS

brain energy metabolism, cerebral cortex, hippocampus, Seahorse XFe96, striatum 


\section{Significance}

Brain mitochondrial dysfunction is implicated in several neurodegenerative diseases and might be linked to inherent regional differences in mitochondrial function. This study reports several differences in the rates of oxygen consumption and ATP synthesis of mitochondria isolated from the mouse cerebral cortex, hippocampus, and striatum. These findings may serve as a foundation for further investigations of the link between regional mitochondrial function and neurodegeneration.

\section{1 | INTRODUCTION}

The mammalian brain is a complex and energy-demanding organ. It is estimated that $70 \%-80 \%$ of the total energy expenditure of the brain is accounted for by processes related to neuronal signaling (Attwell \& Laughlin, 2001; Harris, Jolivet, \& Attwell, 2012). This means that a constant production of energy is crucial for sustaining cerebral function. Mitochondria are cellular structures responsible for the majority of energy production through oxidative phosphorylation, which is the oxygen-dependent production of adenosine- $5^{\prime}$-triphosphate (ATP) (Figure 1a) (Nunnari \& Suomalainen, 2012). Inside the mitochondrial matrix, metabolic substrates are oxidized in the tricarboxylic acid (TCA) cycle, generating energy rich electrons, transported through the reduced carriers $\mathrm{NADH}$ and $\mathrm{FADH}_{2}$. These electrons are subsequently transferred through the electron transport chain (ETC), consisting of distinct protein complexes (I-IV), to generate a proton motive force by pumping protons out of the mitochondrial matrix, which drives the ATP synthase (also known as complex $V$ ) to synthesize ATP (Papa, Petruzzella, \& Scacco, 2007).

A growing body of evidence suggests that brain mitochondrial dysfunction can mediate or accelerate the development of several neurodegenerative diseases (Correia et al., 2012; Johri \& Beal, 2012; Nunnari \& Suomalainen, 2012; Pathak, Berthet, \& Nakamura, 2013). The brain consists of several well-defined areas, each handling different cerebral functions and with distinct susceptibility to neurodegenerative diseases (Camandola \& Mattson, 2017; Masters et al., 2015; Poewe et al., 2017; Ross \& Tabrizi, 2011). For example, Alzheimer's disease primarily affects the cerebral cortex and hippocampus, whereas Huntington's disease manifests predominantly in the striatum (Masters et al., 2015; Ross \& Tabrizi, 2011). Furthermore, mitochondria found throughout the different brain regions and cell types display a large degree of heterogeneity in terms of distribution and efficiency (Dubinsky, 2009; Sonnewald, Hertz, \& Schousboe, 1998). Collectively, these observations may imply that inherent regional differences in mitochondrial function could be the basis of regional susceptibility to brain diseases. An in-depth understanding of regional differences in mitochondrial bioenergetics may elucidate possible mechanisms for the selective neurodegeneration.
Cerebral mitochondrial function can be investigated using several experimental approaches and a common preparation is isolated mitochondria (Clark \& Nicklas, 1970; Lai, Walsh, Dennis, \& Clark, 1977). The use of isolated mitochondria allows a high degree of control and manipulation by circumventing cellular pathways. Previous studies have primarily been performed on isolated mitochondria from the rat brain, due to the larger amount of starting material when compared to mice (Clark \& Nicklas, 1970; Dagani, Marzatico, \& Curti, 1988; Lai et al., 1977; Sims, 1990). However, recent technological advances have allowed high-throughput microplate-based assessment of small quantities of mitochondria, making it possible to run several conditions in parallel (Barros et al., 2018; Connolly et al., 2018; Rogers et al., 2011; Sauerbeck et al., 2011). Here, we apply two such assays to investigate the rate of oxygen consumption and importantly link this to the rate of ATP production, in isolated non-synaptic mitochondria of the mouse brain from three areas often affected by neurodegeneration: the cerebral cortex, hippocampus, and striatum. In addition, using two different mitochondrial fuels, pyruvate and succinate, we dissect regional mitochondrial function mediated through complex I and complex II of the ETC.

\section{2 | METHODS}

\section{1 | Materials}

Pyruvic acid (107360), succinic acid (S3674), malic acid (M1000), adenosine-5'-diphosphate (ADP, A5285), oligomycin A (75351), carbonyl cyanide 4-(trifluoromethoxy)phenylhydrazone (FCCP, C2920), antimycin A (A8674), and rotenone (R8875) were purchased from Sigma-Aldrich (St. Louis, MO, USA). Percoll ${ }^{\text {TM }}$ (17-0891-01) was from GE Healthcare, Uppsala, Sweden. Luciferin-luciferase (11-501) was from BioThema AB (Handen, Sweden) and used according to the manufacturer's instructions. All other reagents were of the purest grade available from regular commercial sources.

\subsection{Animals}

Six male NMRI mice (Envigo, Cambridgeshire, United Kingdom) of 12 weeks of age (weight: $42.2 \mathrm{~g} \pm 3.4 \mathrm{~g}$ ) were housed in a pathogen-free, temperature and humidity-controlled environment at the Department of Drug Design and Pharmacology, University of Copenhagen. The mice were acclimatized for 2 weeks before experiments and were single-housed in individually ventilated cages with free access to chow and water. The experiments were approved by the Danish National Ethics Committee and performed in accordance with the European Convention (ETS 123 of 1986). Mitochondrial function is affected by sex steroids (Gaignard et al., 2017). To avoid introducing this potential confounding variation, all experiments were performed on male mice and hence sex specific aspects of the work could not be evaluated. 


\section{3 | Cerebral mitochondrial isolation}

Isolation of non-synaptic mitochondria from the cerebral cortex, hippocampus, and striatum was performed as described in Chinopoulos, Zhang, Thomas, Ten, and Starkov (2011) with slight modifications. All procedures were performed on ice or at $4^{\circ} \mathrm{C}$. Mice were euthanized by cervical dislocation, decapitated and the brain quickly excised from the cranial vault onto a cooled surface covered by a damp filter paper. The brain was dissected into the three distinct regions: cerebral cortex, hippocampus, and striatum as described by Spijker (2011). Tissue was dissected from both hemispheres with a total dissection time of approximately $3 \mathrm{~min}$. After dissection, the tissue was submerged in isolation buffer (MSHE), containing in $\mathrm{mM}$ : mannitol 210, sucrose 70, HEPES 5, EGTA 1 and 0.5\% BSA (fatty acid free), $\mathrm{pH} 7.2$, and gently homogenized using a Teflon on glass douncer at 500 revolutions/min for 7-8 strokes. The homogenate was centrifuged $(500 \mathrm{~g} \times 5 \mathrm{~min}$ ) to pellet cell remnants. The supernatant was centrifuged $(14.000 \mathrm{~g} \times 10 \mathrm{~min})$ and the pellet re-suspended in $12 \%$ Percoll MSHE solution. This suspension was gently layered on a $21 \%$ Percoll MSHE solution and centrifuged (18.000 $\mathrm{g} \times 15 \mathrm{~min}$ ). The supernatant was discarded and the pellet was washed with two subsequent centrifugations ( $18.000 \mathrm{~g} \times 5 \mathrm{~min}$ and $14.000 \mathrm{~g} \times 5 \mathrm{~min}$ ) by suspending the pellet in MSHE buffer and discarding the supernatant after each centrifugation. The final pellet, containing the isolated mitochondria, was re-suspended and diluted in MSHE and protein amounts were determined using the Bradford protein assay with BSA as standard protein.

\section{4 | Mitochondrial oxygen consumption-Seahorse $\mathrm{XFe} 96$ assay}

The oxygen consumption rate (OCR) of regionally isolated mitochondria was investigated using a Seahorse XFe96 analyzer (Seahorse Biosciences, MA, USA) (luso, Repp, Biagosch, Terrile, \& Prokisch, 2017; Rogers et al., 2011). The isolated mitochondria were diluted in assay buffer (MAS), containing in mM: mannitol 220, sucrose 70 , $\mathrm{KH}_{2} \mathrm{PO}_{4} 10, \mathrm{MgCl}_{2}$ 5, HEPES 2, EGTA 1 and 0.2\% BSA (fatty acid free), $\mathrm{pH}$ 7.2. $25 \mu \mathrm{l}$ of mitochondrial suspension, containing $2 \mu \mathrm{g}$ of protein for the succinate condition and $4 \mu \mathrm{g}$ of protein for the pyruvate/malate condition, were added to a Seahorse 96-well plate and centrifuged $\left(2000 \mathrm{~g} \times 20 \mathrm{~min} \times 4^{\circ} \mathrm{C}\right)$. After centrifugation, $155 \mu \mathrm{l}$ MAS containing pyruvate $(10 \mathrm{mM})$ in combination with malate $(2 \mathrm{mM})$ or succinate $(10 \mathrm{mM})$ and rotenone $(2 \mu \mathrm{M})$ (all final concentrations and $\mathrm{pH}$ 7.2), were added and the wells and the plate was analyzed immediately at $37^{\circ} \mathrm{C}$. Each condition was performed in seven technical replicates for each mitochondrial preparation.

The applied experimental template is known as the mitochondrial coupling assay, investigating the coupling between the ETC and oxidative phosphorylation (Rogers et al., 2011). Each experiment consisted of nine measurements cycles, with intermediate injections of different mitochondrial modulators as depicted in Figure 1b. Each measurement cycle lasted 3 min and was followed by 2 min of mixing and 1 min of rest. Firstly, three baselines measurements were performed before addition of pharmacological compounds. After injection of a compound, 1 min of mixing was performed before the measurement cycle was initiated. The compounds added were (all in final concentrations): ADP (4 mM), oligomycin A $(2.5 \mu \mathrm{g} / \mathrm{ml})$, FCCP $(4 \mu \mathrm{M})$ and antimycin $A(4 \mu M)$. Firstly, ADP was injected, which stimulates ATP synthesis and thus complex $V$-driven respiration. Subsequently, oligomycin $\mathrm{A}$, an inhibitor of complex $\mathrm{V}$, was added to terminate the ADPmediated respiration. The uncoupler FCCP allows protons from the intermembrane space to flow into the matrix (Figure 1a), stimulating uncoupled respiration. Finally, antimycin A was injected to inhibit the flow of electrons from complex III to complex IV, thus leaving only residual non-mitochondrial oxygen consumption, which was measured for three measurement cycles. The Seahorse assay works by forming a transient micro-chamber by bringing oxygen-sensitive probes close to the mitochondria in the wells. Due to this, concentrations of respiratory substrates and mitochondrial modulators may seem high when compared to other mitochondrial assays. The applied amount of mitochondria, concentrations of respiratory substrates, and injected compounds (saturating concentrations) were all verified as optimal in our setup. It was assured that $\mathrm{O}_{2}$ was not depleted during the measurement period and that the $\mathrm{O}_{2}$ levels reached baseline values before the next measurement cycle. Data were collected and extracted using the Wave software (Agilent Technologies, CA, USA).

Absolute OCR is presented as $\mathrm{pmol} \mathrm{O}_{2}$ consumed/min/ $\mathrm{\mu g}$ protein. From the Seahorse experiments nine data points were obtained, denoted (1)-(9) (Figure 1b). Before data analyses, non-mitochondrial respiration was excluded by subtracting point (9) from all individual values. Basal respiration was calculated as an average of point (1), (2), and (3). ADP absolute refers to point (4), proton leak refers to point (5) and FCCP absolute refers to point (6). ADP relative was calculated as (4) divided by (3) * $100 \%$ and FCCP relative was calculated as (6) divided by (3) * $100 \%$. As a general measure of overall mitochondrial function, the respiratory control ratio (RCR) was calculated as point (4) divided by point (5) (Brand \& Nicholls, 2011).

\section{5 | Mitochondrial ATP synthesis-Luciferin- luciferase assay}

The rate of ATP synthesis was measured in the isolated mitochondria with an online luciferin-luciferase coupled luminescence assay using a NOVOstar microplate reader at $28^{\circ} \mathrm{C}$ for optimal luciferinluciferase response (BMG Labtech $\mathrm{GmbH}$, Ortenberg, Germany) (Jakobsen et al., 2018). Mitochondria were diluted in respiration buffer, containing in $\mathrm{mM}$ : sucrose $250, \mathrm{~K}_{2} \mathrm{PO}_{4} 15, \mathrm{MgSO}_{4} 2$, EDTA 0.5 and $0.5 \%$ BSA (fatty acid free), $\mathrm{pH}$ 7.2. $10 \mu \mathrm{l}$ of mitochondrial suspension containing $2.5 \mu \mathrm{g}$ of protein was added to a clear bottom 96-well plate, containing the respiratory substrates: $10 \mathrm{mM}$ pyruvate in combination with $2 \mathrm{mM}$ malate or $10 \mathrm{mM}$ succinate and $2 \mu \mathrm{M}$ rotenone (all final concentrations and $\mathrm{pH}$ 7.2). To inhibit adenylate cyclase activity, all wells also contained $0.2 \mu \mathrm{M} \mathrm{P}^{1}, \mathrm{P}^{5}$ Di(adenosine- $5^{\prime}$ )pentaphosphate pentasodium. A luciferin-luciferase cocktail and hexokinase-purified ADP (final concentration $0.6 \mathrm{mM}$ ) was added to each well and luminescence was assayed 
for $36 \mathrm{~s}$ followed by injection of $9.62 \mu \mathrm{M}$ ATP (final concentration) as internal standard. Each condition was performed in four technical replicates. As a negative control, some wells were added $2.5 \mu \mathrm{g} / \mathrm{ml}$ oligomycin A, to inhibit ATP synthesis (data not shown). Mitochondrial ATP synthesis is presented as pmol ATP produced/ $\mathrm{min} / \mu \mathrm{g}$ protein. As a measure of mitochondrial efficacy, that is, ATP production in relation to $\mathrm{O}_{2}$ consumption, an $A T P / \mathrm{O}_{2}$ ratio was calculated as the rate of ATP production divided by the ADP-stimulated OCR determined by Seahorse analyses.

\section{6 | Mitochondrial membrane potential- TMRM assay}

To monitor the mitochondrial membrane potential $\left(\Delta \Psi_{m}\right)$, tetramethylrhodamine methyl ester (TMRM) was added ( $1 \mu \mathrm{M}$, final concentration) to $1 \mu \mathrm{g}$ of regionally isolated mitochondria and measured in quench mode (530 nm excitation/590 nm emission) in a NOVOstar microplate reader at $28^{\circ} \mathrm{C}$ (BMG Labtech $\mathrm{GmbH}$, Ortenberg, Germany) (Jakobsen et al., 2018; Nicholls \& Ward, 2000). After a stable baseline was reached (30 min), oligomycin A (5 $\mu \mathrm{g} / \mathrm{ml} \mu \mathrm{M}$, final

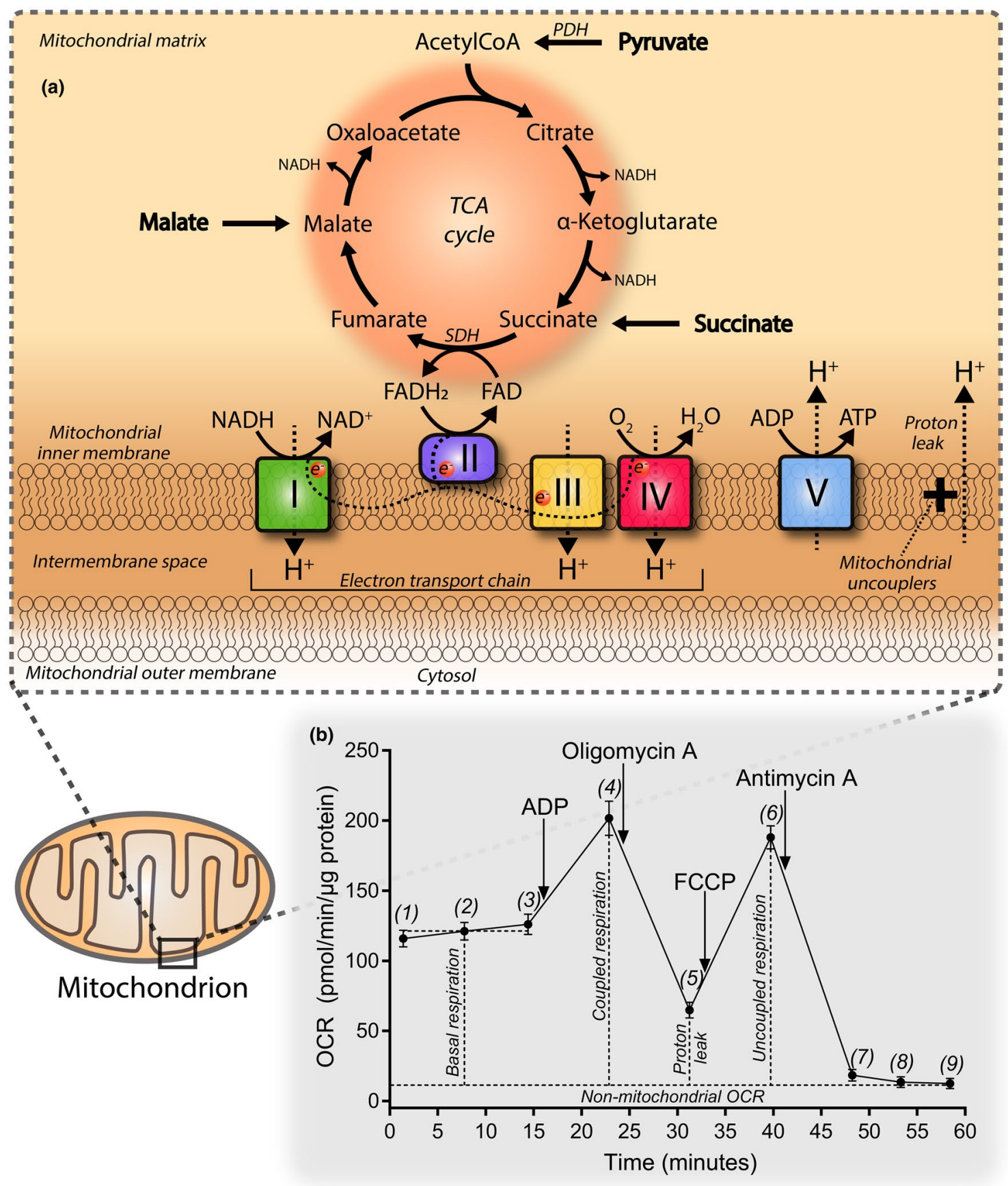


FIGURE 1 Mitochondrial bioenergetics. (a) Mitochondrial overview. Mitochondria are double membrane enclosed structures, consisting of an inner and an outer membrane, separating the cytosol, the intermembrane space and the mitochondrial matrix. In the tricarboxylic acid (TCA) cycle, located inside the mitochondrial matrix, substrates can be oxidized to produce the reduced electron carriers NADH and $\mathrm{FADH}_{2}$. In the inner mitochondrial membrane, protein complexes (designated I-IV) are embedded, collectively known as the electron transport chain (ETC). Electrons from NADH are transferred to complex I, whereas electrons from $\mathrm{FADH}_{2}$ are transferred to complex II. The enzyme converting succinate to fumarate, succinate dehydrogenase (SDH), is an integral part of complex II. The flow of electrons through the ETC, drives the expulsion of protons $\left(\mathrm{H}^{+}\right)$, through complex I, III, and IV, out of the mitochondrial matrix into the intermembrane space, creating a proton motive force. In complex IV, electrons are neutralized by $\mathrm{O}_{2}$. The proton gradient, generated by the ETC, can be utilized by the ATP synthase (complex V) to convert ADP into ATP. The entire process with oxidative metabolism of substrates, ETC activity, oxygen consumption, and ATP synthesis is known as oxidative phosphorylation. To investigate mitochondrial function in isolated mitochondria, pyruvate in combination with malate or succinate can be provided as substrates. Pyruvate is converted into acetylCoA by activity of pyruvate dehydrogenase (PDH). The provided malate will form oxaloacetate needed to oxidize acetylCoA units in the TCA cycle. Since most of the reactions in the TCA cycle form NADH, oxidation of pyruvate in combination with malate, is mediated primarily via complex I. Succinate can also be provided as a metabolic substrate and will generate $\mathrm{FADH}_{2}$ directly for the ETC via SDH activity. Since oxaloacetate is a powerful inhibitor of SDH, it is customary to add rotenone, a complex I inhibitor, to the media, to ensure that succinate respiration is not inhibited and only reflects complex II activity. In coupled mitochondria it is the level of ATP that decides the activity of the ETC, that is, there is a coupling between ATP synthesis and ETC activity. However, protons can also cross the inner mitochondrial matrix independently of complex $\mathrm{V}$, which is known as the proton leak. Certain compounds known as mitochondrial uncouplers, can greatly increase this flow of protons into the mitochondrial matrix bypassing complex $\mathrm{V}$, which will induce uncoupled respiration. (b) Seahorse coupling assay. The Seahorse coupling assay can be used to assess critical mitochondrial parameters including coupled and uncoupled oxygen consumption rates (OCR) and proton leak. Firstly, three basal measurements are performed (1-3) in the presence of the respiratory substrates. ADP is then added to the mitochondria which will induce coupled respiration by stimulating ATP synthesis (4). To inhibit coupled respiration, oligomycin A, a complex $\mathrm{V}$ inhibitor is added to the mitochondria (5). FCCP is a mitochondrial uncoupler, allowing protons to cross the inner mitochondrial membrane and induce uncoupled respiration, circumventing complex V (6). Finally, antimycin A, an inhibitor of complex III, is added to assess non-mitochondrial respiration (7-9). Arrows indicate addition of the respective compounds. The data shown are from cerebral cortical mitochondria provided succinate (in the presence of rotenone) as respiratory substrate, mean \pm standard deviation, $n=6$

concentration) was added to assess the mitochondrial proton leak (Figure 1b). Measurements were performed in a clear bottom 96well plate, in the same respiration buffer used for the ATP synthesis assay, containing $10 \mathrm{mM}$ pyruvate in combination with $2 \mathrm{mM}$ malate or $10 \mathrm{mM}$ succinate and $2 \mu \mathrm{M}$ rotenone, both conditions with $0.2 \mu \mathrm{M}$ $\mathrm{P}^{1}, \mathrm{P}^{5}$-Di(adenosine- $5^{\prime}$ )pentaphosphate pentasodium (all final concentrations and $\mathrm{pH}$ 7.2). Traces of mitochondrial membrane potential are presented as $\Delta \Psi_{m}\left(F_{0} / F\right)$, in which data points were normalized to the baseline levels obtained within the first 5 min of measurements.

\section{7 | Study design and handling of data}

This study was designed as an exploratory study with no allocation of subjects into experimental groups, hence no blinding or randomization were applied. Data shown represent means \pm standard deviations (unless other is noted), with individual data points plotted as well. The data are based on six individual experiments (biological replicates, i.e., from individual animals), except for the ATP measurements with pyruvate/malate where only four data points were obtained due to an experimental defect in the enzymatic assay. In some instances, negative values were obtained due to methodological errors. All negative values were removed prior to analysis. If the amount of negative values exceeded more than half of the technical replicates the data point was omitted (one incidence: RCR and proton leak with pyruvate/malate). Sample sizes were estimated based on a pilot study and previous experiments from which it has been observed that the variability and validity of data is optimal as indicated by standard errors generally below $10 \%$ of the mean values.
Data were inspected visually and is assumed to follow normal distribution. Data were analyzed using repeated measures analysis of variance (ANOVA) with Tukey's post hoc test in GraphPad Prism 7. $p$-Values $<0.05$ was considered significant and is indicated with a single asterisk. Means, standard deviations, number of biological replicates and all statistical analyzes are reported in Tables 1 and 2.

\section{3 | RESULTS}

\section{1 | Pyruvate/malate-Complex I-driven respiration and ATP synthesis}

Pyruvate is the end-product of glycolysis and a major mitochondrial fuel (Sonnewald et al., 1998). To investigate NADH-linked, that is, complex I-mediated, respiration and ATP synthesis, isolated mitochondria were provided pyruvate and malate as respiratory substrates. The provided malate enables formation of oxaloacetate needed for oxidation of acetylCoA, generated through activity of pyruvate dehydrogenase (PDH), in the TCA cycle (Figure 1a).

From the basal respiration of the isolated mitochondria, it was observed that hippocampal mitochondria exhibited the lowest OCR compared to the cerebral cortical $\left(F_{2,10}=88.17, p<0.0001\right)$ and striatal $\left(F_{2,10}=88.17, p<0.0001\right)$ mitochondria (Figure 2a). Furthermore, the OCR of cerebral cortical mitochondria was lower than striatal mitochondria $\left(F_{2,10}=88.17, p=0.0015\right)$, which had the highest basal OCR. When ADP was added, it was again observed that hippocampal mitochondria had a lower OCR when compared to cerebral cortical $\left(F_{2,10}=70.03, p<0.0001\right)$ and striatal $\left(F_{2,10}=70.03\right.$, 


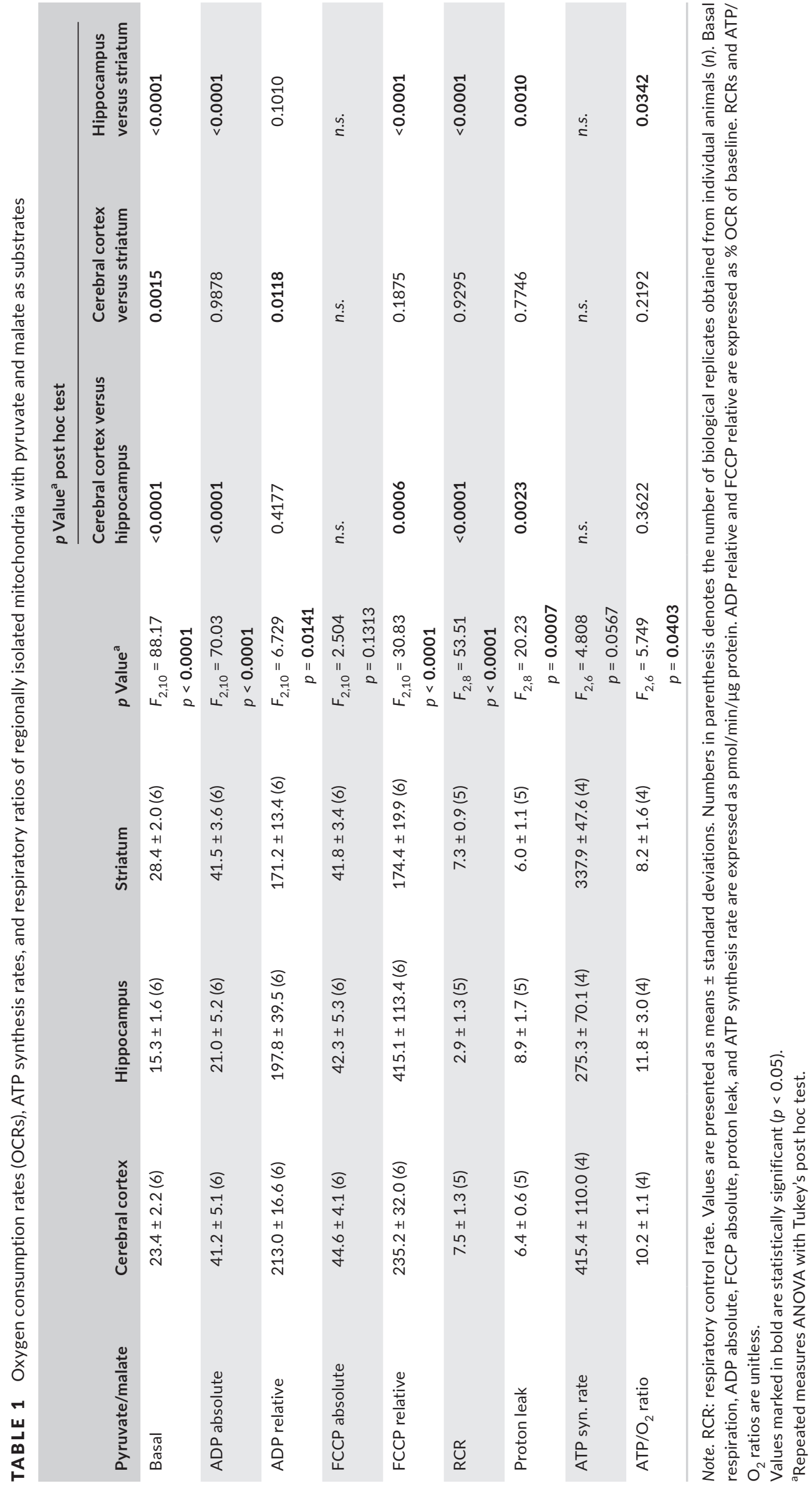




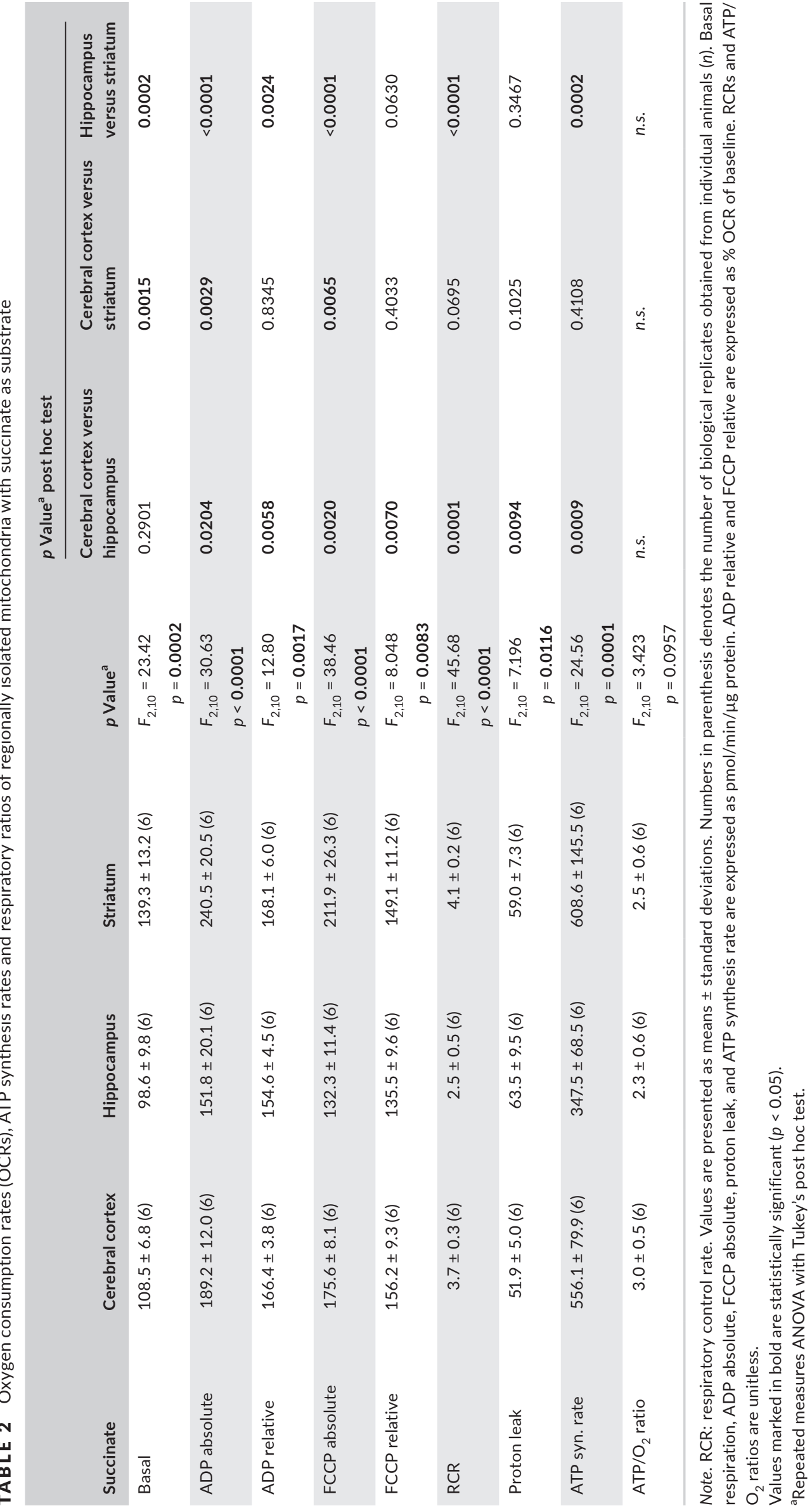



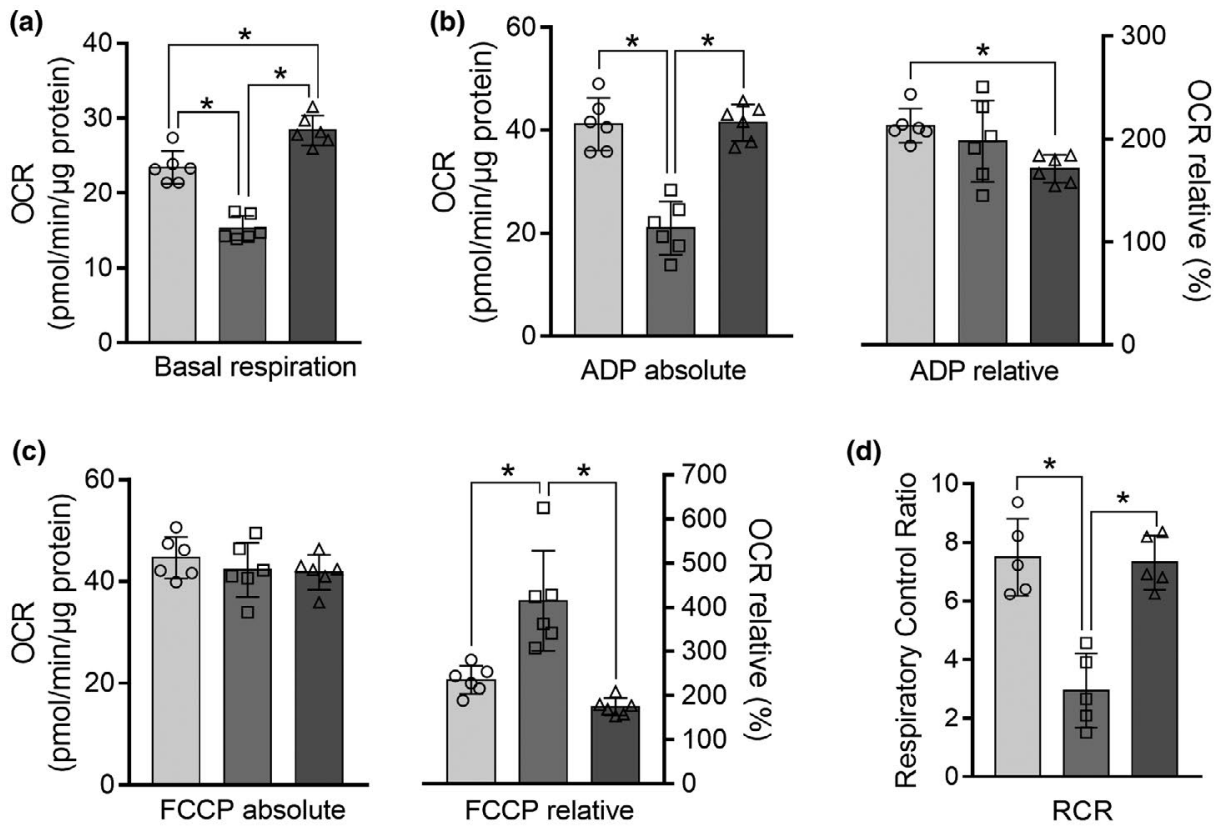

(d)
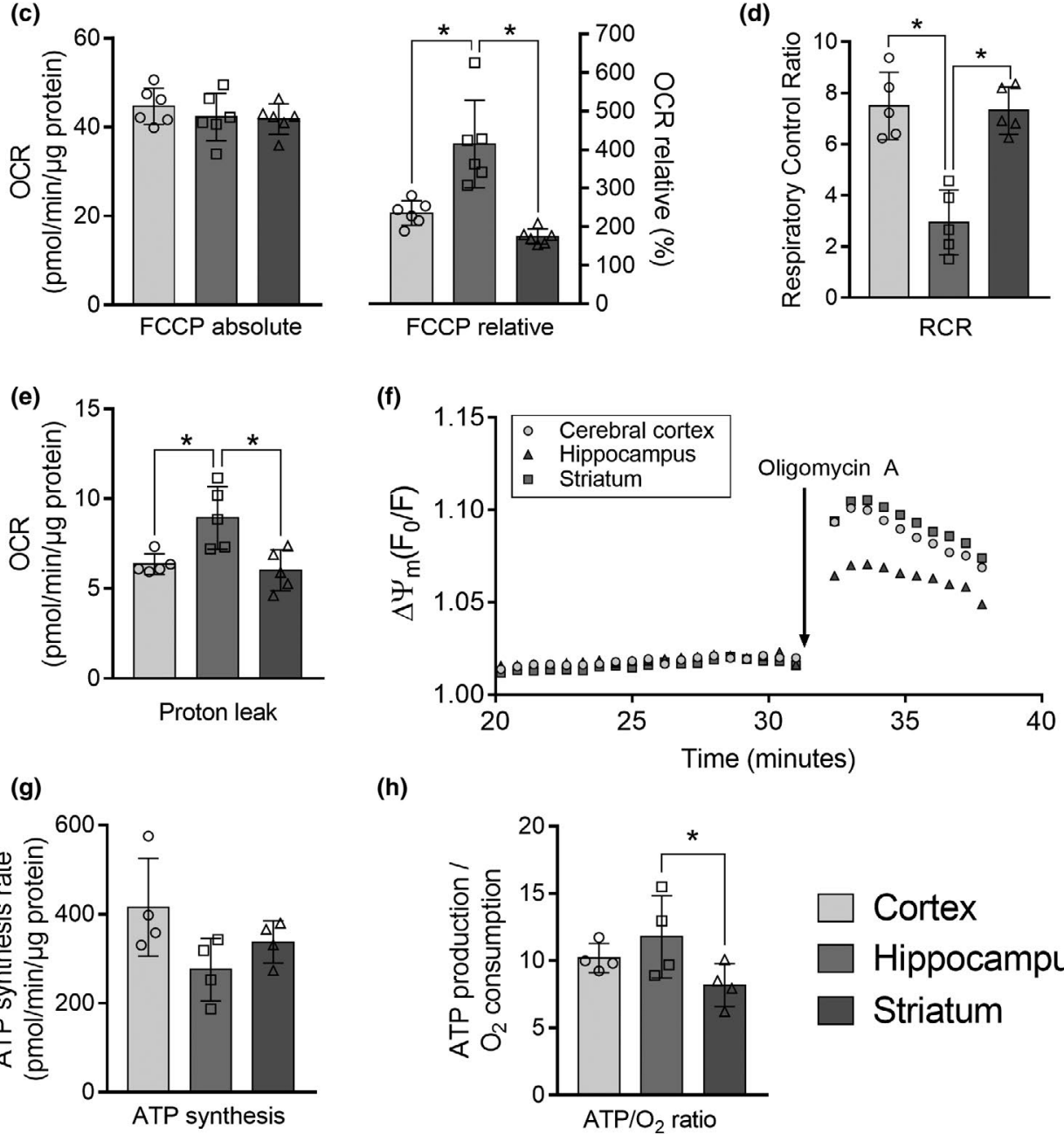

(h)

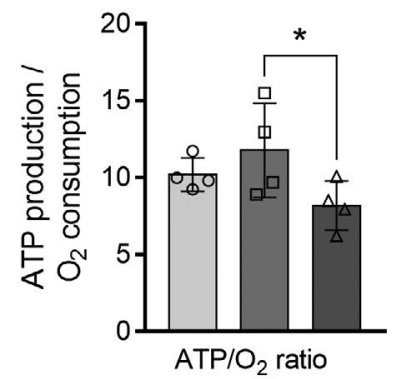

\section{Cortex}

Hippocampus

Striatum

FIGURE 2 Hippocampal mitochondria exhibits a lower metabolic rate accompanied by a large potential for uncoupled respiration when provided pyruvate and malate. Oxygen consumption rates (OCRs) and ATP synthesis rates of isolated mitochondria of the mouse cerebral cortex (open circles), hippocampus (open squares), and striatum (open triangles) with pyruvate (10 mM) and malate (2 mM) as substrates. (a) Basal OCR. (b) ADP-stimulated (coupled) OCR, absolute (left) and relative (right). (c) FCCP-stimulated (uncoupled) OCR, absolute (left) and relative (right). (d) Respiratory control ratio (RCR). (e) Proton leak. (f): Traces of mitochondrial membrane potential (means only). (g) ATP synthesis rate. (h) ATP $/ \mathrm{O}_{2}$ ratio. Results are presented as means \pm standard deviations, $n=4-6$ obtained from individual animals. Repeated measures ANOVA, Tukey's post hoc test, ${ }^{*} p<0.05$

$p<0.0001$ ) mitochondria (Figure 2b). Due to the larger basal respiration of the striatal mitochondria, a significant decrease in relative OCR, in response to ADP, was observed when compared to cerebral cortical mitochondria $\left(F_{2,10}=6.729, p=0.0118\right)$.

Uncoupled respiration, that is, respiration circumventing complex $\mathrm{V}$, induced by FCCP showed no significant differences between the three regions (Figure 2c). However, the hippocampal mitochondria showed the greatest relative increase in OCR in response to FCCP when compared to mitochondria of the cerebral cortex $\left(F_{2,10}=30.83, p=0.0006\right)$ and striatum $\left(F_{2,10}=30.83\right.$, $p<0.0001$ ). To assess the overall mitochondrial function, the RCR was calculated. It was observed that hippocampal mitochondria had 
a significantly reduced RCR when compared to cerebral cortical $\left(F_{2,8}=53.51, p<0.0001\right)$ and striatal $\left(F_{2,8}=53.51, p<0.0001\right) \mathrm{mi}-$ tochondria (Figure $2 \mathrm{~d}$ ). This difference results from a higher proton leak of the hippocampal mitochondria, that is, residual respiration in the presence of the complex $V$ inhibitor oligomycin $A$, which was observed when compared to cerebral cortical $\left(F_{2,8}=20.23, p=0.0023\right)$ and striatal $\left(F_{2,8}=20.23, p=0.0010\right)$ mitochondria (Figure 2e). The elevated hippocampal proton leak was furthermore validated by a lower membrane potential of hippocampal mitochondria in the presence of oligomycin A, when compared to cerebral cortical and striatal mitochondria (Figure 2f).

No changes were found in the ATP production rate of the three regions (Figure $2 \mathrm{~g}$ ). To further evaluate the mitochondrial efficiency of substrate oxidation, that is, how much ATP is produced relative to oxygen consumption, a ratio between ATP production and oxygen consumption was calculated (Figure $2 \mathrm{~h}$ ). Here, it was observed that striatal mitochondria exhibited a slightly reduced $A T P / O_{2}$ ratio when compared to hippocampal mitochondria $\left(F_{2,6}=5.749, p=0.0342\right)$.

\section{2 | Succinate-Complex II-driven respiration and ATP synthesis}

Complex II-driven respiration and ATP synthesis can be assessed by providing the mitochondria with succinate as respiratory substrate. Succinate is converted into fumarate by activity of succinate dehydrogenase $(\mathrm{SDH})$, which is part of complex II and hereby directly provide $\mathrm{FADH}_{2}$ to the ETC (Figure 1a). To ensure that succinate oxidation reflects complex II, that is, $\mathrm{FADH}_{2}$-mediated respiration, the complex I inhibitor rotenone was included in the respiration buffer. Rotenone inhibits the oxidation of $\mathrm{NADH}$ to $\mathrm{NAD}^{+}$, which will block the conversion of malate to oxaloacetate as this reaction reduces $\mathrm{NAD}^{+}$to $\mathrm{NADH}$ (Figure 1a). Since oxaloacetate is a powerful inhibitor of SDH, the addition of rotenone will increase the oxidation of succinate (Papa, Lofrumento, Paradies, \& Quagliariello, 1969; Wojtczak, Wojtczak, \& Ernster, 1969).

When the regionally isolated mitochondria were provided succinate, it was observed that striatal mitochondria exhibited a significantly increased basal OCR in comparison to both cerebral cortical $\left(F_{2,10}=23.42, p=0.0015\right)$ and hippocampal $\left(F_{2,10}=23.42\right.$, $p=0.0002$ ) mitochondria (Figure 3a). ADP stimulation also led to the largest increase in absolute OCR in striatal mitochondria when compared to mitochondria of the cerebral cortex $\left(F_{2,10}=30.63\right.$, $p=0.0029)$ and hippocampus $\left(F_{2,10}=30.63, p<0.0001\right)$ (Figure $\left.3 b\right)$. Furthermore, the hippocampal mitochondria exhibited a decreased OCR when compared to cerebral cortical mitochondria $\left(F_{2,10}=30.63\right.$, $p=0.0204)$. The differences in relative OCR upon ADP stimulation were of a much smaller magnitude. However, it was observed that the relative OCR of the hippocampal mitochondria again was decreased when compared to mitochondria of the cerebral cortex $\left(F_{2,10}=12.80, p=0.0058\right)$ and striatum $\left(F_{2,10}=12.80, p=0.0024\right)$.

When challenged with FCCP, mitochondria of the striatum showed an elevated OCR when compared to cerebral cortical $\left(F_{2,10}=38.64, p=0.0065\right)$ and hippocampal $\left(F_{2,10}=38.64\right.$, $p<0.0001$ ) mitochondria, and the OCR of hippocampal mitochondria were furthermore decreased when compared to cerebral cortical mitochondria $\left(F_{2,10}=38.64, p=0.0020\right)$ (Figure 3c). The relative OCR, during FCCP stimulation, showed that the OCR of hippocampal mitochondria was significantly smaller when compared to mitochondria of the cerebral cortex $\left(F_{2,10}=8.048, p=0.0070\right)$ and the same trend was present when compared to striatal mitochondria $\left(F_{2,10}=8.048\right.$, $p=0.0630$ ). As observed for the pyruvate/malate condition, mitochondria of the hippocampus had a significantly reduced RCR when compared to cerebral cortical $\left(F_{2,10}=45.68, p=0.0001\right)$ and striatal $\left(F_{2,10}=45.68, p<0.0001\right)$ mitochondria (Figure 3d). The decreased RCR was again mediated by an increased hippocampal proton leak, which was higher when compared to cerebral cortical mitochondria $\left(F_{2,10}=7.196, p=0.0094\right)$, but not striatal mitochondria (Figure 3e). However, from measurements of the mitochondrial membrane potential, it was observed that hippocampal mitochondria exhibited a lower membrane potential in the presence of oligomycin A, indicative of an elevated proton leak, when compared to both mitochondria of the cerebral cortex and striatum (Figure 3f).

Hippocampal mitochondria had the lowest rate of ATP production compared to cerebral cortical $\left(F_{2,10}=24.56, p=0.0009\right)$ and striatal $\left(F_{2,10}=24.56, p=0.0002\right)$ mitochondria (Figure $3 g$ ). However, the ATP production was balanced by oxygen consumption, signified by equal ATP/O $\mathrm{O}_{2}$ ratios for all regions (Figure $3 \mathrm{~h}$ ).

\section{4 | DISCUSSION}

Here, we investigated the rates of oxygen consumption and ATP synthesis, in isolated mitochondria of the mouse cerebral cortex, hippocampus, and striatum using different respiratory substrates. We provide functional evidence of heterogeneity in mitochondrial energetics between the three brain regions investigated. When pyruvate and malate were provided as substrates, hippocampal mitochondria exhibited the lowest basal and coupled OCR, however, with comparable efficiency of ATP synthesis to the other regions. Hippocampal mitochondria also exhibited a larger proton leak and elevated relative OCR in response to FCCP, showing a large capacity for uncoupled respiration. When the complex II-linked substrate succinate was provided, striatal mitochondria exhibited the highest respiration and ATP synthesis rate, whereas hippocampal mitochondria had the lowest. However, equal ATP/O $\mathrm{O}_{2}$ ratios between the regions suggests similar mitochondrial efficiency of succinate oxidation.

\subsection{Mitochondrial isolation and function}

In this report, cerebral mitochondria were isolated using a Percoll density gradient, allowing separation of non-synaptic mitochondria from other cellular components, including myelin and cellular debris, but also unwanted metabolic active entities such as synaptosomes (Chinopoulos et al., 2011; Sims, 1990). Isolation of mitochondria allows tight experimental control and the possibility of direct manipulation, but may compromise other physiological factors, such as 

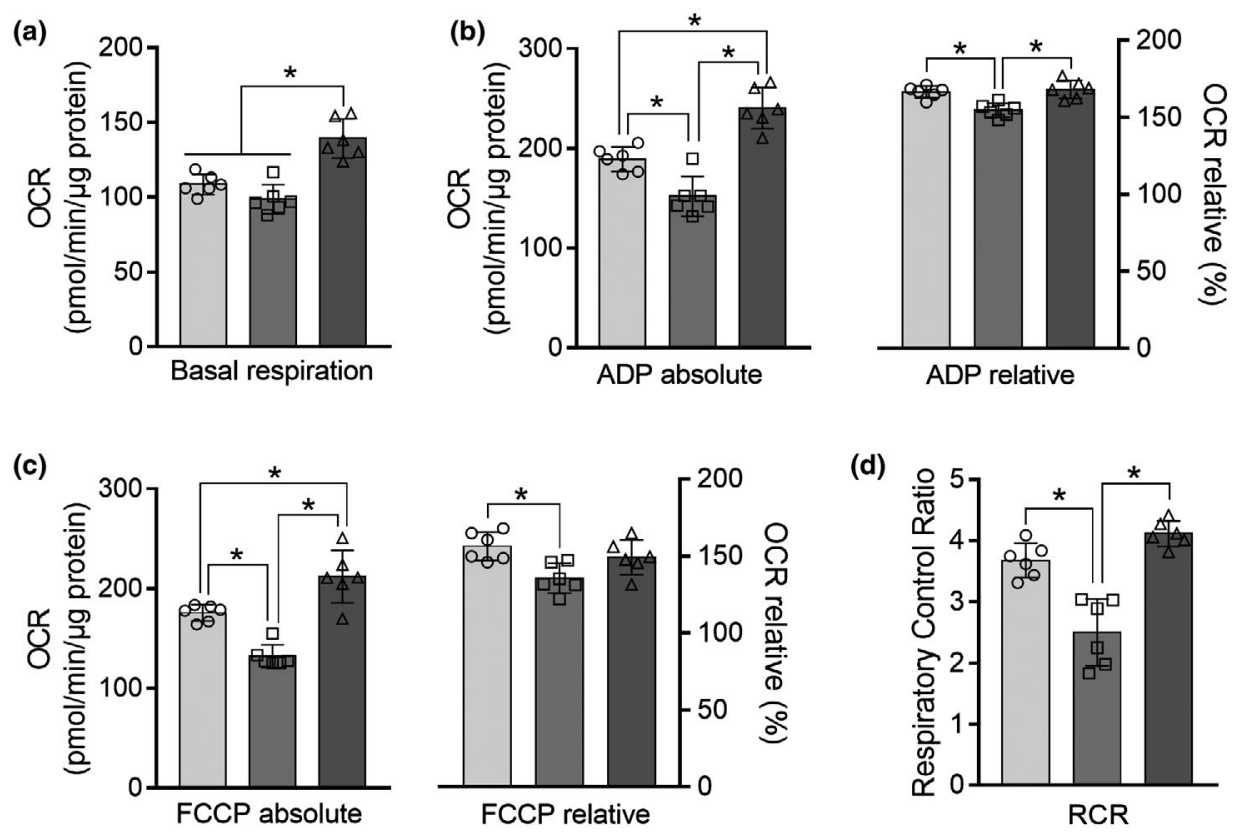

(d)
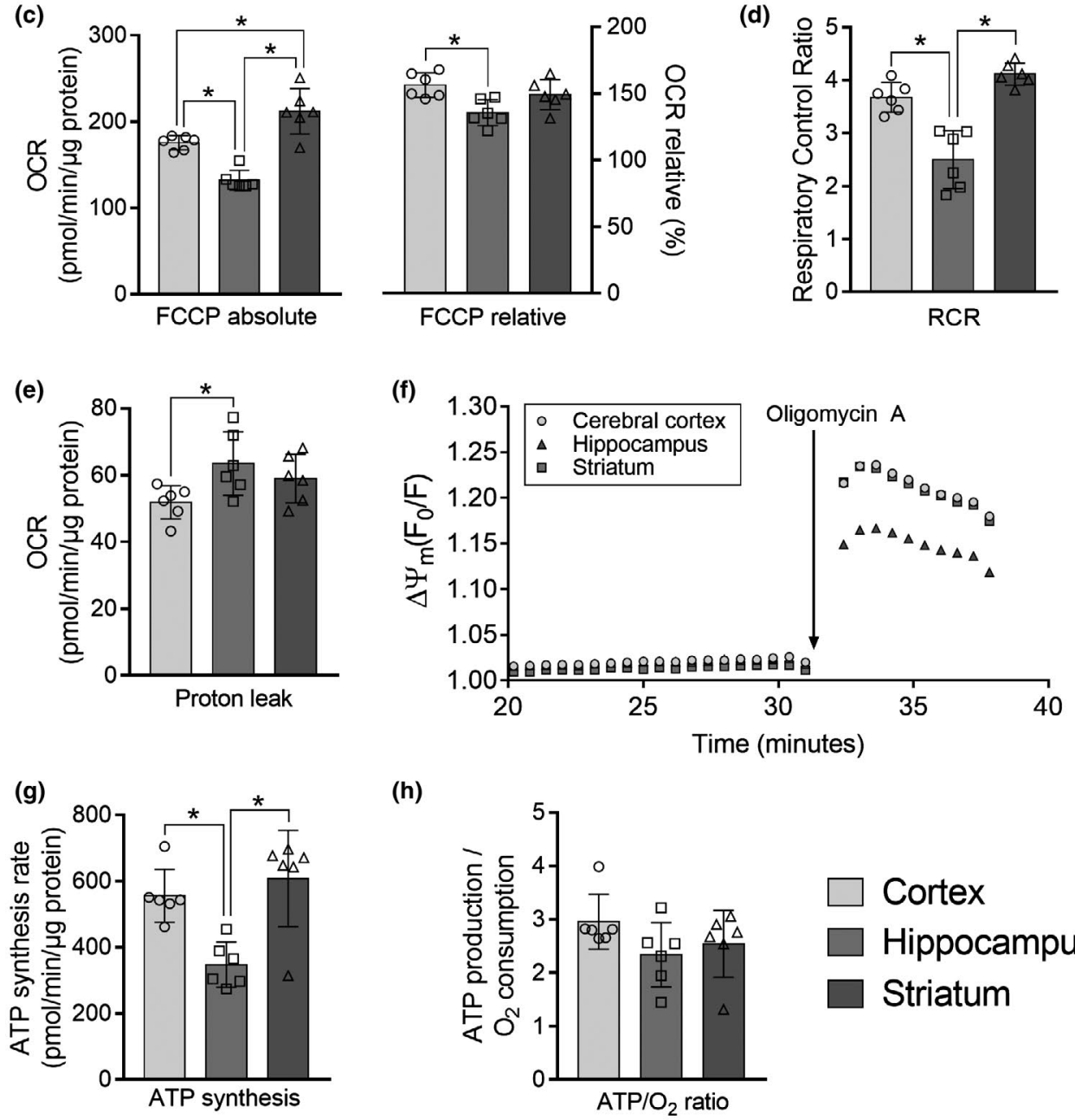

(h)

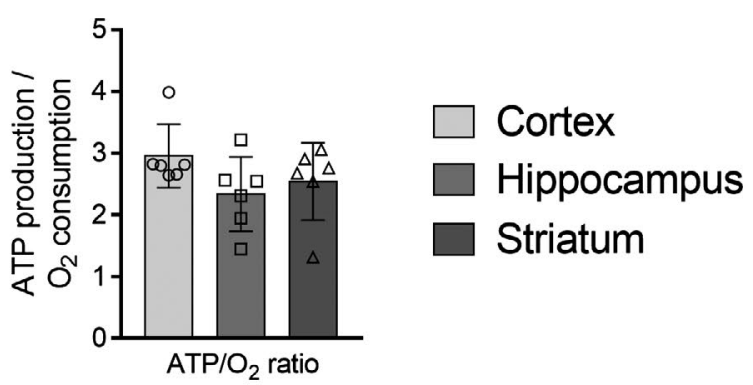

FIGURE 3 Striatal mitochondria display elevated basal and stimulated oxygen consumption when provided succinate as respiratory substrate. Isolated mitochondria from mouse cerebral cortex (open circles), hippocampus (open squares), and striatum (open triangles) were provided succinate $(10 \mathrm{mM})$, in the presence of the complex I inhibitor rotenone $(2 \mu \mathrm{M})$, as respiratory substrate. (a) Basal OCR. (b) ADPstimulated (coupled) OCR, absolute (left) and relative (right). (c) FCCP-stimulated (uncoupled) OCR, absolute (left) and relative (right). (d) Respiratory control ratio (RCR). (e) Proton leak. (f) Traces of mitochondrial membrane potential (means only). (g) ATP synthesis rate. (h) ATP/ $\mathrm{O}_{2}$ ratio. Results are presented as means \pm standard deviations, $n=6$ obtained from individual animals. Repeated measures ANOVA, Tukey's post hoc test, ${ }^{*} p<0.05$

structure, intra-cellular connections, and signaling pathways (Brand \& Nicholls, 2011; Picard, Taivassalo, Gouspillou, \& Hepple, 2011). To assess the integrity of isolated mitochondria, the RCR is a commonly applied relative measure that describes overall mitochondrial function (Brand \& Nicholls, 2011). We obtained RCRs values in the range of 3-7 with pyruvate/malate and 2-4 with succinate as substrate. Compared to the literature, these values signify well-functioning mitochondria, validating the quality of our preparation (Burtscher, Zangrandi, Schwarzer, \& Gnaiger, 2015; Cardoso, Santos, Seica, \& Moreira, 2010; Czerniczyniec et al., 2015; Dagani et al., 1988; Lai et al., 1977; Sauerbeck et al., 2011; Sims, 1990; Varela, Schwartz, \& Horvath, 2016). We observed that hippocampal mitochondria exhibited a larger proton leak, with both substrate conditions, resulting in reduced $\mathrm{RCR}$ values for this region. This is an important notion, as 
the choice of substrate should not alter the RCR (Brand \& Nicholls, 2011). However, since succinate oxidation does not contribute to the generation of the proton motive force through complex I of the ETC, the RCR values often tends to be lower when compared to $\mathrm{NADH}$ linked substrates, which was also the case in the present study (Brand \& Nicholls, 2011). The isolation technique used for this study yields non-synaptic mitochondria originating from both neurons and glial cells. The Percoll isolation yields a mitochondrial fraction with a high purity (Chinopoulos et al., 2011; Sims, 1990), however, some contamination of membrane proteins and synaptosomes will still be present. It should therefore be noted that the isolated mitochondrial fractions are derived from heterogeneous cellular populations and might exhibit different degrees of contamination, which potentially could have influenced the results.

\section{2 | Regional differences in mitochondrial OCR and ATP synthesis rate}

Only few other studies, using Seahorse technology to assess mitochondrial function in regionally isolated brain mitochondria, have been performed. A study by Sauerbeck et al. showed that mitochondria isolated from the rat cerebral cortex, hippocampus, and striatum exhibited equal coupled OCRs with pyruvate/malate, and the same uncoupled OCRs with succinate in the presence of rotenone (Sauerbeck et al., 2011). Interestingly, the authors also reported a trend toward a larger residual respiration in the presence of oligomy$\operatorname{cin} \mathrm{A}$, that is, proton leak, of striatal and hippocampal mitochondria (Sauerbeck et al., 2011). Here, we observed an increased proton leak in isolated hippocampal mitochondria of the mouse brain, however, this was not the case in the striatal mitochondria. Another recent study, likewise performed in rats, showed that regional mitochondrial function fluctuates with age (Pandya, Royland, MacPhail, Sullivan, \& Kodavanti, 2016). The authors reported that cerebral cortical, striatal, and hippocampal mitochondria, isolated from young rats, exhibited comparable bioenergetics profiles, however, particularly hippocampal mitochondrial function declined with increasing age (Pandya et al., 2016). A relevant extension of this study could include assessment of how regional mitochondrial function in mice is related to age.

We found that mitochondria of the hippocampus, generally consumed oxygen and synthesized ATP at lower rates, when compared to mitochondria of the other two regions. These observations of low hippocampal oxygen consumption is in line with several other reports of isolated mitochondria (Burtscher et al., 2015; Cardoso et al., 2010; Czerniczyniec et al., 2015; Dagani et al., 1988; Sims, 1990; Varela et al., 2016) and brain homogenates (Sims, Finegan, \& Blass, 1986; Sims \& Pulsinelli, 1987). However, it was also observed that hippocampal mitochondria exhibited comparable ATP/O $\mathrm{O}_{2}$ ratios to the other regions, suggesting an overall general lower mitochondrial activity in this region (Dagani et al., 1988). A striking finding of this study is the hippocampal capacity of uncoupled respiration when provided pyruvate and malate. Even though both the basal and ADP-induced OCR of the hippocampal mitochondria were lower, the absolute uncoupled respiration was equal across the regions, leading to a much larger relative OCR increase. This elevated hippocampal uncoupling capacity has also been observed in hippocampal synaptosomes fueled by glucose (Lee et al., 2013), and shows that hippocampal mitochondria possess the same capacity for maximal uncoupled respiration as the other regions, which is not active during coupled respiration. We also observed that the RCR of hippocampal mitochondria were significantly lower when compared to the other regions, due to an elevated proton leak across the inner mitochondrial membrane. The proton leak constitute a large fraction of the resting mitochondrial metabolic rate but the mechanisms and functions are still unclear (Jastroch, Divakaruni, Mookerjee, Treberg, \& Brand, 2010). The proton leak is affected by the mitochondrial membrane composition, including fatty acid composition and expression of transporters and proteins (Brand, Chien, Ainscow, Rolfe, \& Porter, 1994; Jastroch et al., 2010; Stuart, Cadenas, Jekabsons, Roussel, \& Brand, 2001). One group of proteins, the uncoupling proteins (UCPs), allows protons to cross the inner mitochondrial membrane and are involved in the proton leak (Jastroch et al., 2010). The functions of the UCPs in the brain are still unclear, but they influence mitochondrial function, calcium handling, and production of reactive oxygen species (Andrews, Diano, \& Horvath, 2005). Further studies have to reveal if UCPs could be linked to the observed differences in hippocampal mitochondrial function and possibly neurodegenerative diseases.

Mitochondria isolated from the striatum exhibited the largest basal OCR, when provided both pyruvate/malate and succinate. Furthermore, the absolute OCR of striatal mitochondria, when provided succinate, was larger than mitochondria of the cerebral cortex, which was not the case when using pyruvate and malate as respiratory substrates. Several recent studies have reported elevations in both coupled and uncoupled OCRs of striatal mitochondria when provided succinate as substrate, which is in line with our results (Burtscher et al., 2015; Pandya et al., 2016; Subramaniam, Vergnes, Franich, Reue, \& Chesselet, 2014). A general hypothesis on the pathogenesis of Huntington's disease, a disease primarily affecting the striatum, is that the mitochondria of the striatum are more sensitive to metabolic insults and therefore more susceptible to neurodegeneration (Browne \& Beal, 2004; Damiano, Galvan, Deglon, \& Brouillet, 2010; Mochel \& Haller, 2011). Interestingly, systemic injections with the SDH inhibitor, 3-nitropropionic acid, leads to selective lesions in the striatum, suggesting that striatal mitochondria are vulnerable to inhibition of SDH (Beal et al., 1993; Brouillet et al., 1995). Our results of elevated basal respiration and increased capacity of succinate oxidation of striatal mitochondria are in line with this and support that SDH impairments may be a sensitive target of neurodegeneration in the striatum.

\section{3 | Complex I- and II-mediated OCR and ATP synthesis rate}

When comparing the applied respiratory substrate conditions, pyruvate/malate versus succinate, reflecting complex I and complex II-linked respiration, respectively, the absolute differences in OCRs are striking. The OCRs were found to be approximately 
fourfold higher for succinate when compared to pyruvate/malate for all regions. This observation is in accordance with previous reports (Clark \& Nicklas, 1970; luso et al., 2017; Rogers et al., 2011), and is explained by succinate providing electrons for the ETC with only one enzymatic reaction through $\mathrm{SDH}$ with subsequent $\mathrm{FADH}_{2}$ generation. Pyruvate, on the other hand, must undergo several consecutive enzymatic steps in order to provide NADH for the ETC. However, the ATP synthesis rate was not increased to the same extent as the OCR, which became apparent by the ATP/O $/ \mathrm{O}_{2}$ ratios, which were threefold reduced for succinate when compared to pyruvate/malate. This notion is in line with the classic phosphate/ oxygen (P/O or ADP/O) ratios, describing how many moles ATP are synthesized/0.5 moles $\mathrm{O}_{2}$ consumed (Hinkle \& Yu, 1979). The standard $\mathrm{P} / \mathrm{O}$ ratios are estimated to be 2.5 for $\mathrm{NADH}$-linked substrates (such as pyruvate in combination with malate) and 1.5 for succinate (Hinkle, 2005; Hinkle \& Yu, 1979). We found that oxidative phosphorylation on pyruvate/malate in combination were roughly three times more efficient than succinate, when OCRs were compared to the rates of ATP synthesis. This result might seem excessive when compared to the established $\mathrm{P} / \mathrm{O}$ ratios, however, our calculations are based on the combination of two different assays, making the direct comparison difficult as the experimental prerequisites are different.

\subsection{Conclusions}

This study reveals clear differences in OCR and ATP synthesis rates, mediated through complex I and complex II of the ETC, in isolated mitochondria from cerebral cortex, hippocampus, and striatum of the mouse brain. We found that mitochondrial efficiency, that is, ATP synthesis $/ \mathrm{O}_{2}$ consumed, was comparable between the three regions. Hippocampal mitochondria exhibited a larger proton leak, whereas striatal mitochondria had an elevated basal respiration and increased capacity for succinate oxidation. This study underlines the importance of investigating cerebral mitochondrial function regionally, as regional differences may by masked when multiple regions are pooled. Furthermore, we advocate to asses OCR in combination with ATP synthesis rate, as either one alone can be misleading.

\section{DECLARATION OF TRANSPARENCY}

The authors, reviewers, and editors affirm that in accordance to the policies set by the Journal of Neuroscience Research, this manuscript presents an accurate and transparent account of the study being reported and that all critical details describing the methods and results are present.

\section{ACKNOWLEDGMENTS}

JVA gratefully acknowledge the financial support of the Scholarship of Peter \& Emma Thomsen.

\section{CONFLICT OF INTEREST}

The authors declare no potential conflict of interests.

\section{AUTHOR CONTRIBUTIONS}

Methodology, J.V.A., E.J., H.S.W., and B.I.A.; Investigation, J.V.A. and E.J.; Formal Analysis, J.V.A. and E.J.; Writing - Original Draft, J.V.A. All authors have contributed with critical intellectual content and have approved the final manuscript.

\section{DATA ACCESSIBILITY}

Data can be provided via e-mail upon request.

\section{ORCID}

Jens V. Andersen (iD https://orcid.org/0000-0001-8767-2675

Emil Jakobsen (iD https://orcid.org/0000-0001-6290-4222

Helle S. Waagepetersen (iD https://orcid.org/0000-0003-4583-8379

Blanca I. Aldana iD https://orcid.org/0000-0002-5027-1218

\section{REFERENCES}

Andrews, Z. B., Diano, S., \& Horvath, T. L. (2005). Mitochondrial uncoupling proteins in the CNS: In support of function and survival. NatureReviews Neuroscience, 6(11), 829-840. https://doi. org/10.1038/nrn1767

Attwell, D., \& Laughlin, S. B. (2001). An energy budget for signaling in the grey matter of the brain. Journal of Cerebral Blood Flow and Metabolism: Official Journal of the International Society of Cerebral Blood Flow and Metabolism, 21(10), 1133-1145. https://doi. org/10.1097/00004647-200110000-00001

Barros, L. F., Bolanos, J. P., Bonvento, G., Bouzier-Sore, A. K., Brown, A., Hirrlinger, J., ... Weber, B. (2018). Current technical approaches to brain energy metabolism. Glia, 66(6), 1138-1159. https://doi. org/10.1002/glia.23248

Beal, M. F., Brouillet, E., Jenkins, B. G., Ferrante, R. J., Kowall, N. W., Miller, J. M., ... Hyman, B. T. (1993). Neurochemical and histologic characterization of striatal excitotoxic lesions produced by the mitochondrial toxin 3-nitropropionic acid. The Journal of Neuroscience: The Official Journal of the Society for Neuroscience, 13(10), 4181-4192. https://doi.org/10.1523/JNEUROSCI.13-10-04181.1993

Brand, M. D., Chien, L. F., Ainscow, E. K., Rolfe, D. F., \& Porter, R. K. (1994). The causes and functions of mitochondrial proton leak. Biochimica et Biophysica Acta, 1187(2), 132-139. https://doi. org/10.1016/0005-2728(94)90099-X

Brand, M. D., \& Nicholls, D. G. (2011). Assessing mitochondrial dysfunction in cells. The Biochemical Journal, 435(2), 297-312. https://doi. org/10.1042/BJ20110162

Brouillet, E., Hantraye, P., Ferrante, R. J., Dolan, R., Leroy-Willig, A., Kowall, N. W., \& Beal, M. F. (1995). Chronic mitochondrial energy impairment produces selective striatal degeneration and abnormal choreiform movements in primates. Proceedings of the National Academy of Sciences of the United States of America, 92(15), 71057109. https://doi.org/10.1073/pnas.92.15.7105

Browne, S. E., \& Beal, M. F. (2004). The energetics of Huntington's disease. Neurochemical Research, 29(3), 531-546. https://doi. org/10.1023/B:NERE.0000014824.04728.dd 
Burtscher, J., Zangrandi, L., Schwarzer, C., \& Gnaiger, E. (2015). Differences in mitochondrial function in homogenated samples from healthy and epileptic specific brain tissues revealed by highresolution respirometry. Mitochondrion, 25, 104-112. https://doi. org/10.1016/j.mito.2015.10.007

Camandola, S., \& Mattson, M. P. (2017). Brain metabolism in health, aging, and neurodegeneration. The EMBO Journal, 36(11), 1474-1492.

Cardoso, S., Santos, M. S., Seica, R., \& Moreira, P. I. (2010). Cortical and hippocampal mitochondria bioenergetics and oxidative status during hyperglycemia and/or insulin-induced hypoglycemia. Biochimica et Biophysica Acta, 1802(11), 942-951. https://doi.org/10.1016/j. bbadis.2010.07.001

Chinopoulos, C., Zhang, S. F., Thomas, B., Ten, V., \& Starkov, A. A. (2011). Isolation and functional assessment of mitochondria from small amounts of mouse brain tissue. Methods in Molecular Biology (Clifton, NJ), 793, 311-324.

Clark, J. B., \& Nicklas, W. J. (1970). The metabolism of rat brain mitochondria. Preparation and characterization. The Journal of Biological Chemistry, 245(18), 4724-4731.

Connolly, N. M. C., Theurey, P., Adam-Vizi, V., Bazan, N. G., Bernardi, P., Bolanos, J. P., ... Prehn, J. H. M. (2018). Guidelines on experimental methods to assess mitochondrial dysfunction in cellular models of neurodegenerative diseases. Cell Death and Differentiation, 25(3), 542-572. https://doi.org/10.1038/s41418-017-0020-4

Correia, S. C., Santos, R. X., Perry, G., Zhu, X., Moreira, P. I., \& Smith, M. A. (2012). Mitochondrial importance in Alzheimer's, Huntington's and Parkinson's diseases. Advances in Experimental Medicine and Biology, 724, 205-221. https://doi. org/10.1007/978-1-4614-0653-2_16

Czerniczyniec, A., La Padula, P., Bustamante, J., Karadayian, A. G., LoresArnaiz, S., \& Costa, L. E. (2015). Mitochondrial function in rat cerebral cortex and hippocampus after short- and long-term hypobaric hypoxia. Brain Research, 1598, 66-75. https://doi.org/10.1016/j. brainres.2014.12.018

Dagani, F., Marzatico, F., \& Curti, D. (1988). Oxidative metabolism of nonsynaptic mitochondria isolated from rat brain hippocampus: A comparative regional study. Journal of Neurochemistry, 50(4), 1233-1236. https://doi.org/10.1111/j.1471-4159.1988.tb10598.x

Damiano, M., Galvan, L., Deglon, N., \& Brouillet, E. (2010). Mitochondria in Huntington's disease. Biochimica et Biophysica Acta, 1802(1), 5261. https://doi.org/10.1016/j.bbadis.2009.07.012

Dubinsky, J. M. (2009). Heterogeneity of nervous system mitochondria: Location, location, location! Experimental Neurology, 218(2), 293307. https://doi.org/10.1016/j.expneurol.2009.05.020

Gaignard, P., Liere, P., Therond, P., Schumacher, M., Slama, A., \& Guennoun, R. (2017). Role of sex hormones on brain mitochondrial function, with special reference to aging and neurodegenerative diseases. Frontiers in Aging Neuroscience, 9, 406. https://doi. org/10.3389/fnagi.2017.00406

Harris, J. J., Jolivet, R., \& Attwell, D. (2012). Synaptic energy use and supply. Neuron, 75(5), 762-777. https://doi.org/10.1016/j. neuron.2012.08.019

Hinkle, P. C. (2005). P/O ratios of mitochondrial oxidative phosphorylation. Biochimica et Biophysica Acta, 1706(1-2), 1-11. https://doi. org/10.1016/j.bbabio.2004.09.004

Hinkle, P. C., \& Yu, M. L. (1979). The phosphorus/oxygen ratio of mitochondrial oxidative phosphorylation. The Journal of Biological Chemistry, 254(7), 2450-2455.

luso, A., Repp, B., Biagosch, C., Terrile, C., \& Prokisch, H. (2017). Assessing mitochondrial bioenergetics in isolated mitochondria from various mouse tissues using seahorse XF96 analyzer. Methods in Molecular Biology (Clifton, NJ), 1567, 217-230. https://doi. org/10.1007/978-1-4939-6824-4_13

Jakobsen, E., Lange, S. C., Andersen, J. V., Desler, C., Kihl, H. F., Hohnholt, M. C., ... Bak, L. K. (2018). The inhibitors of soluble adenylate cyclase 2-OHE, $\mathrm{KH} 7$, and bithionol compromise mitochondrial ATP production by distinct mechanisms. Biochemical Pharmacology, 155, 92-101. https://doi.org/10.1016/j.bcp.2018.06.023

Jastroch, M., Divakaruni, A. S., Mookerjee, S., Treberg, J. R., \& Brand, M. D. (2010). Mitochondrial proton and electron leaks. Essays in Biochemistry, 47, 53-67. https://doi.org/10.1042/bse0470053

Johri, A., \& Beal, M. F. (2012). Mitochondrial dysfunction in neurodegenerative diseases. The Journal of Pharmacology and Experimental Therapeutics, 342(3), 619-630. https://doi.org/10.1124/ jpet.112.192138

Lai, J. C., Walsh, J. M., Dennis, S. C., \& Clark, J. B. (1977). Synaptic and non-synaptic mitochondria from rat brain: Isolation and characterization. Journal of Neurochemistry, 28(3), 625-631. https://doi. org/10.1111/j.1471-4159.1977.tb10434.x

Lee, D. Y., Xun, Z., Platt, V., Budworth, H., Canaria, C. A., \& McMurray, C. T. (2013). Distinct pools of non-glycolytic substrates differentiate brain regions and prime region-specific responses of mitochondria. PLoS ONE, 8(7), e68831. https://doi.org/10.1371/journal. pone.0068831

Masters, C. L., Bateman, R., Blennow, K., Rowe, C. C., Sperling, R. A., \& Cummings, J. L. (2015). Alzheimer's disease. Nature Reviews Disease Primers, 1, 15056. https://doi.org/10.1038/nrdp.2015.56

Mochel, F., \& Haller, R. G. (2011). Energy deficit in Huntington disease: Why it matters. The Journal of Clinical Investigation, 121(2), 493-499. https://doi.org/10.1172/JCl45691

Nicholls, D. G., \& Ward, M. W. (2000). Mitochondrial membrane potential and neuronal glutamate excitotoxicity: Mortality and millivolts. Trends in Neurosciences, 23(4), 166-174. https://doi.org/10.1016/ S0166-2236(99)01534-9

Nunnari, J., \& Suomalainen, A. (2012). Mitochondria: In sickness and in health. Cell, 148(6), 1145-1159. https://doi.org/10.1016/j. cell.2012.02.035

Pandya, J. D., Royland, J. E., MacPhail, R. C., Sullivan, P. G., \& Kodavanti, P. R. (2016). Age- and brain region-specific differences in mitochondrial bioenergetics in Brown Norway rats. Neurobiology of Aging, 42, 25-34. https://doi.org/10.1016/j.neurobiolaging.2016.02.027

Papa, S., Lofrumento, N. E., Paradies, G., \& Quagliariello, E. (1969). Mechanism of inhibition by uncouples of succinate oxidation in isolated mitochondria. Biochimica et Biophysica Acta, 180(1), 35-44. https://doi.org/10.1016/0005-2728(69)90191-1

Papa, S., Petruzzella, V., \& Scacco, S.2007. 1.5 electron transport. Structure, redox-coupled protonmotive activity, and pathological disorders of respiratory chain complexes. In A. Lajtha, G. E. Gibson, \& G. A. Dienel (Eds.), Handbook of neurochemistry and molecular neurobiology: Brain energetics integration of molecular and cellular processes (pp. 93-118). Boston, MA: Springer, US.

Pathak, D., Berthet, A., \& Nakamura, K. (2013). Energy failure: Does it contribute to neurodegeneration? Annals of Neurology, 74(4), 506516. https://doi.org/10.1002/ana.24014

Picard, M., Taivassalo, T., Gouspillou, G., \& Hepple, R. T. (2011). Mitochondria: Isolation, structure and function. The Journal of Physiology, 589(Pt 18), 4413-4421. https://doi.org/10.1113/ jphysiol.2011.212712

Poewe, W., Seppi, K., Tanner, C. M., Halliday, G. M., Brundin, P., Volkmann, J., ... Lang, A. E. (2017). Parkinson disease. NatureReviews Disease Primers, 3, 17013. https://doi.org/10.1038/nrdp.2017.13

Rogers, G. W., Brand, M. D., Petrosyan, S., Ashok, D., Elorza, A. A., Ferrick, D. A., \& Murphy, A. N. (2011). High throughput microplate respiratory measurements using minimal quantities of isolated mitochondria. PLoS ONE, 6(7), e21746. https://doi.org/10.1371/journal. pone.0021746

Ross, C. A., \& Tabrizi, S. J. (2011). Huntington's disease: From molecular pathogenesis to clinical treatment. The Lancet Neurology, 10(1), 8398. https://doi.org/10.1016/S1474-4422(10)70245-3

Sauerbeck, A., Pandya, J., Singh, I., Bittman, K., Readnower, R., Bing, G., \& Sullivan, P. (2011). Analysis of regional brain mitochondrial bioenergetics and susceptibility to mitochondrial inhibition utilizing a 
microplate based system. Journal of Neuroscience Methods, 198(1), 36-43. https://doi.org/10.1016/j.jneumeth.2011.03.007

Sims, N. R. (1990). Rapid isolation of metabolically active mitochondria from rat brain and subregions using Percoll density gradient centrifugation. Journal of Neurochemistry, 55(2), 698-707. https://doi. org/10.1111/j.1471-4159.1990.tb04189.x

Sims, N. R., Finegan, J. M., \& Blass, J. P. (1986). Effects of postdecapitative ischemia on mitochondrial respiration in brain tissue homogenates. Journal of Neurochemistry, 47(2), 506-511. https://doi. org/10.1111/j.1471-4159.1986.tb04530.x

Sims, N. R., \& Pulsinelli, W. A. (1987). Altered mitochondrial respiration in selectively vulnerable brain subregions following transient forebrain ischemia in the rat. Journal of Neurochemistry, 49(5), 1367-1374. https://doi.org/10.1111/j.1471-4159.1987.tb01001.x

Sonnewald, U., Hertz, L., \& Schousboe, A. (1998). Mitochondrial heterogeneity in the brain at the cellular level. Journal of Cerebral Blood Flow and Metabolism: Official Journal of the International Society of Cerebral Blood Flow and Metabolism, 18(3), 231-237. https://doi. org/10.1097/00004647-199803000-00001

Spijker, S. (2011). Dissection of rodent brain regions. In K. W. Li (Ed.), Neuroproteomics (pp. 13-26). Totowa, NJ: Humana Press.

Stuart, J. A., Cadenas, S., Jekabsons, M. B., Roussel, D., \& Brand, M. D. (2001). Mitochondrial proton leak and the uncoupling protein 1 homologues. Biochimica et Biophysica Acta, 1504(1), 144-158. https:// doi.org/10.1016/S0005-2728(00)00243-7

Subramaniam, S. R., Vergnes, L., Franich, N. R., Reue, K., \& Chesselet, M. F. (2014). Region specific mitochondrial impairment in mice with widespread overexpression of alpha-synuclein. Neurobiology of Disease, 70, 204-213. https://doi.org/10.1016/j.nbd.2014.06.017

Varela, L., Schwartz, M. L., \& Horvath, T. L. (2016). Mitochondria controlled by UCP2 determine hypoxia-induced synaptic remodeling in the cortex and hippocampus. Neurobiology of Disease, 90, 68-74. https://doi.org/10.1016/j.nbd.2016.01.004

Wojtczak, L., Wojtczak, A. B., \& Ernster, L. (1969). The inhibition of succinate dehydrogenase by oxalacetate. Biochimica et Biophysica Acta, 191(1), 10-21. https://doi.org/10.1016/0005-2744(69)90310-6

\section{SUPPORTING INFORMATION}

Additional supporting information may be found online in the Supporting Information section at the end of the article.

Transparent Science Questionnaire for Authors.

How to cite this article: Andersen JV, Jakobsen E, Waagepetersen HS, Aldana BI. Distinct differences in rates of oxygen consumption and ATP synthesis of regionally isolated non-synaptic mouse brain mitochondria. J Neuro Res. 2019;97:961-974. https://doi.org/10.1002/jnr.24371 